\title{
Multilocus Lod Scores in Large Pedigrees: Combination of Exact and Approximate Calculations
}

\author{
Liping Tong Elizabeth Thompson \\ Department of Statistics, University of Washington, Seattle, Wash., USA
}

\section{Key Words}

Meiosis indicator $\cdot$ Block-Gibbs MCMC $\cdot$ Haplotype update $\cdot$ Multiple meiosis update

\begin{abstract}
To detect the positions of disease loci, lod scores are calculated at multiple chromosomal positions given trait and marker data on members of pedigrees. Exact lod score calculations are often impossible when the size of the pedigree and the number of markers are both large. In this case, a Markov Chain Monte Carlo (MCMC) approach provides an approximation. However, to provide accurate results, mixing performance is always a key issue in these MCMC methods. In this paper, we propose two methods to improve MCMC sampling and hence obtain more accurate lod score estimates in shorter computation time. The first improvement generalizes the block-Gibbs meiosis (M) sampler to multiple meiosis (MM) sampler in which multiple meioses are updated jointly, across all loci. The second one divides the computations on a large pedigree into several parts by conditioning on the haplotypes of some 'key' individuals. We perform exact calculations for the descendant parts where more data are often available, and combine this information with sampling of the hidden variables in the ancestral parts. Our approaches are expected to be most useful for data on a large pedigree with a lot of missing data.
\end{abstract}

Copyright $\odot 2007$ S. Karger AG, Basel

\section{Introduction}

In this paper we develop improved methods for accurate estimation of lod scores for genetic linkage analyses [1], using data jointly at multiple genetic markers on members of extended pedigrees with substantial missing information. To map the genes underlying complex diseases, the usual strategy has been to first localize genes to regions on a scale of centiMorgans (cM) using data on pedigrees. Fine-scale mapping using associations between genotype and phenotype can then identify candidate genes. A recent example is the study of age-related macular degeneration (AMD) [2].

The development of genotyping technology and publicly available information on human genetic variation such as that provided by the HapMap project [3] have made possible the direct testing of associations between genotype and phenotype on a genome-wide scale. Such genome-wide association studies (GWA) raise the possibility of identifying disease-related genes from large samples of unrelated individuals, which are much more easily obtained than are pedigree data. However, it remains to be determined how far GWA studies can replace pedigree linkage analyses. As yet, GWA studies have provided conflicting results in comprehensive nationwide studies of Parkinson disease [4-9] and of obesity [10-13]. Problems of population structure and of genetic heterogeneity, at both the locus and allelic level, impact GWA severely but

\section{KARGER}

Fax +4161306 1234 E-Mail karger@karger.ch www.karger.com (c) 2007 S. Karger AG, Basel

0001-5652/08/0653-0142\$24.50/0

Accessible online at:

www.karger.com/hhe
Liping Tong

Department of Statistics, University of Washington

Box 354322

Seattle, WA $98195-4322$ (USA)

Tel. +1 206543 8292, Fax +1 206685 7419, E-Mail tong@stat.washington.edu 
have little impact on pedigree analyses. Further, the power of linkage analysis is substantially increased by joint analysis of marker data at multiple polymorphic markers observed on members of extended pedigrees ascertained for multiple affected individuals [14]. Thus it remains important to develop computationally efficient methods for obtaining accurate lod scores using such data.

To obtain linkage lods scores, we assume availability of data on the genotypes at multiple linked genetic marker loci $\left(Y_{M}\right)$ and trait characteristics $\left(Y_{T}\right)$ of some subset of the members of extended pedigrees. Genotyping error is not considered in this paper. The genetic marker model, including the genetic map of the marker loci and the population frequencies of marker alleles, is assumed known. We also consider a single-locus model for the trait data $Y_{T}$. The parameter of interest is then the location $\gamma$ of the trait locus on the chromosome of the markers, with $\gamma=$ $\infty$ denoting that the trait locus is not on this chromosome. The lod score is the log-likelihood statistic

$$
\begin{aligned}
\operatorname{lod}(\gamma) & =\log _{10}(L(\gamma) / L(\infty)) \\
& =\log _{10}\left(P_{\gamma}\left(Y_{T}, Y_{M}\right) / P P_{\bullet}\left(Y_{T}, Y_{M}\right)\right) \\
& =\log _{10}\left(P_{\gamma}\left(Y_{T} \mid Y_{M}\right) / P\left(Y_{T}\right)\right)
\end{aligned}
$$

since, in the absence of linkage, trait data $Y_{T}$ and marker data $Y_{M}$ are independent. On extended pedigrees with a substantial proportion of missing observations, a major challenge remains the computation of this lod score [15]. Note that the subscript $\gamma$ is dropped when a probability, such as $P\left(Y_{T}\right)$, does not depend on the location of trait locus.

When exact computation is infeasible or impractical, Markov chain Monte Carlo (MCMC) methods provide a way to estimate the lod score $[16,17]$. Where there are multiple genetic marker loci, both exact and Monte Carlo methods use some form of meiosis indicators [18] or inheritance vectors [19] to achieve the computation. The inheritance of DNA at any locus can be specified by binary meiosis indicators $S_{i j}$, with $S_{i j}=0$ or 1 as in meiosis $i$ at locus $j$ the maternal or paternal DNA of the parent is transmitted to the offspring. Here $i=1, \ldots, m$ indexes the meioses in the pedigree. We assume that the linked marker loci $j=1, \ldots, n$ are ordered along the chromosome. Let $S_{M}$ denote the meiosis indicators relating to all the markers. Then the probability required for the lod score (1) may be written

$$
P_{\gamma}\left(Y_{T} \mid Y_{M}\right)=\sum_{S_{M}} P_{\gamma}\left(Y_{T} \mid S_{M}\right) P\left(S_{M} \mid Y_{M}\right)
$$

The form of equation (2) shows the challenge for exact computation when $m$ and $n$ are both large; $S_{M}$ consists of $m n$ binary indicators. Equation (2) also shows how Monte Carlo estimation may be achieved through sampling $S_{M}$ conditionally on $Y_{M}$ and averaging the resulting values of $P_{\gamma}\left(Y_{T} \mid S_{M}\right)$ for each $\gamma$ of interest.

There are many ways to sample the components of $S_{M}$ conditionally on $Y_{M}$ using MCMC, but among the simplest and most effective are block Gibbs samplers. The locus (or L) sampler [20] jointly resamples $\left\{S_{i j} ; i=1, \ldots, m\right\}$ successively over loci $j$. The meiosis (or M) sampler jointly resamples $\left\{S_{i j} ; j=1, \ldots, n\right\}$ successively over meioses $i$. These two block-Gibbs samplers may be combined to form the LM-sampler [21] which has been the mainstay of our MCMC lod score estimation approaches [22]. The LM-sampler, together with the estimation approach indicated by equation (2), forms the basis of the MORGAN [23] program lm_markers. In comparisons with other software, the $l m$ _markers programs has been found to be quite competitive for lod score estimation on extended pedigrees [24]. We therefore take $l m \_$markers (MORGAN V2.8.1) as the base-point for comparison of the improved MCMC methods presented in this paper. Our new programs are also implemented in the MORGAN package.

In this paper, we describe two ways in which MCMC sampling may be improved in order to obtain more accurate lod score estimates more efficiently. Our first improvement is a generalization of the block-Gibbs meiosis (M) sampler in which multiple meioses are updated jointly, across all loci. The simplest proposal is to update some randomly chosen subset of $k$ meioses, which can be achieved in time of order $k 2^{k}$ using the factored Hidden Markov Model (HMM) method [25]. Alternative proposals are to update specific subsets, such as the two meioses of an individual, the maternal and/or paternal meioses in a sibship, or the meioses of a 3-generation pedigree segment. Where the number of such meioses is too large for simple computation a restricted sampling of such updates has been implemented, similar to that of Thomas et al. [26]. These proposals have been implemented in the program $l m \_$multiple released in version 2.8.2 of MORGAN package [23].

The second method augments the latent state space with the haplotypes of a few 'key' individuals. Sobel and Lange [27] describe two sets of latent variables, descent graphs and descent states. Descent graphs are equivalent to the meiosis indicators $S_{i j}$ which define the descent of DNA at every locus $j$. Descent states specify in addition the haplotypes of founders: the alleles carried on each founder chromosome. The founder allelic types and the meiosis indicators together determine the genotypes of 
all individuals. Thus use of descent states makes computations simpler, but the much larger and more constrained space impairs the mixing performance of the MCMC. Our approach specifies the haplotypic states not of the founders but of key pedigree members who divide the pedigree. Segments of the pedigree are then independent conditional on these haplotypes, permitting either exact computation and independent realizations or MCMC methods to be performed on each segment and the results combined. Both the multiple-meiosis sampling and the augmentation with haplotypes of key individuals are implemented in the program lm_haplotype within the framework of the MORGAN version 2.8 package [23]. The program $l m \_h a p l o t y p e$ is not yet publicly released.

In this paper we first provide the details of these generalizations of our MCMC methods. We then compare the performance of the three MORGAN [23] programs Im_markers, Im_multiple and lm_haplotype, using simulated data at 10 linked markers on a 52-member pedigree. In our simulations and in the paper, we use sex-averaged genetic maps, but this is for ease of presentation only. As with all MORGAN programs [23], these three programs can equally use gender-specific maps. On an extended pedigree, with a substantial portion of missing data, and when marker loci are tightly linked, the $l m \_$markers program can perform poorly. We show how $l m \_$multiple improves estimation of a multipoint lod score, and $l m_{-}$haplotype provides further improvement. For comparison with exact results using VITESSE [28], we use just 4 markers on the full 52-member pedigree. We also compare the results for all 10 markers on a 14 -member subset of the pedigree, using MERLIN [29]. We show that, using our new programs, MCMC is both an accurate and a computationally efficient approach to computation of multipoint lod scores.

\section{Methods}

Meiosis Indicators, Recombination, and Genetic Maps

We first review the probability model and conditional independence structure of the meiosis indicators $S_{i j}$. This structure underlies all multilocus computations and sampling methods. At any single locus $j$, the meiosis indicators $S_{i j}, i=1, \ldots, m$ are independent. However, for each meiosis $i$, the $S_{i j}, j=1, \ldots, n$ are dependent. In meiosis $i$, recombination occurs between two loci $j$ and $l$ if $S_{i j} \neq S_{i l}$. That is, the genes segregating to the offspring are from different grandparents. At any two loci $j$ and $l$, the pairwise distribution of $\left(S_{i j}, S_{i l}\right)$ is determined by the recombination rate $\theta_{j l}$ between the two loci. That is,

$$
P\left(S_{i j} \neq S_{i l}\right)=\theta_{j l} \text {, for each } i=1, \ldots, m \text { and } 0 \leq \theta_{j l} \leq 1 / 2 \text {. }
$$

For loci that are close together on a chromosome, $\theta_{j l}$ is close to 0 . For independently segregating loci, such as loci on different chromosomes, $\theta_{j l}=1 / 2$. Although, in reality and in our software, recombination rates may differ between male and female meioses, for simplicity we use sex-averaged rates in this paper.

To define the joint distribution of $S_{i j}$ over $j=1, \ldots, n$, an additional assumption is required. In this paper, we assume absence of genetic interference so that $\left\{S_{i j}\right\}_{j=1, \ldots, n}$ are independent Markov chains $(i=1, \ldots, m)$. In this case, the Haldane map function [30] provides the conversion between the genetic distance between $j$ and $l$ and the recombination rate $\theta_{j l}$. Let $S_{. j}=\left(S_{1 j}, \ldots, S_{m j}\right)$ denote the meiosis indicators and $Y_{. j}$ denote the observed marker genotype data at locus $j$ over the whole pedigree. At locus $j$, conditional on meiosis indicators $S_{. j}$, the observed data $Y_{. j}$ are independent of observed data and meiosis indicators at other loci.

This hidden Markov structure permits use of the factored HMM method [25] to obtain the forward cumulative probabilities $\alpha_{j}\left(S_{. j}\right)=P\left(S_{. j} \mid Y_{.1}, \ldots, Y_{. j}\right)$ for $j=1, \ldots, n$ in time of order $m n 2^{m}$. Then $S_{._{n}}$ may be resampled from $\alpha_{n}\left(S_{.}\right)$. For $j=n-1, \ldots, 1, S_{. j}$ may be successively resampled from $P\left(S_{\cdot j} \mid S_{\cdot j+1}, Y_{\cdot 1}, \ldots, Y_{\cdot j}\right)$, which may be written as

$$
\begin{aligned}
P\left(S_{\cdot j} \mid S_{\cdot j+1}, Y_{\cdot 1}, \ldots, Y_{\cdot j}\right) & =\frac{P\left(S_{\cdot j}, S_{\cdot j+1} \mid Y_{\cdot 1}, \ldots, Y_{\cdot j}\right)}{\sum_{S_{\cdot j}} P\left(S_{\cdot j}, S_{\cdot j+1} \mid Y_{\cdot 1}, \ldots, Y_{\cdot j}\right)} \\
& =\frac{P\left(S_{\cdot j+1} \mid S_{\cdot j}\right) \alpha_{j}\left(S_{\cdot j}\right)}{\sum_{S_{. j}} P\left(S_{\cdot j+1} \mid S_{\cdot j}\right) \alpha_{j}\left(S_{\cdot j}\right)},
\end{aligned}
$$

since $S_{. j+1}$ is independent of $Y_{\cdot 1}, \ldots, Y_{\cdot j}$ conditional on $S_{. j}$ due to hidden Markov structure.

\section{Multiple Meiosis Sampler}

Except on small pedigrees, the number of meioses $m$ is too large for the above exact forward computation and backwards sampling to be feasible. Thus, only a small subset of the total set of meioses can be sampled jointly. MCMC procedures resample a subset of the indicators $S_{i j}$ conditional on the data and current values of the remaining indicators. An MCMC iteration (or scan) consists of a sequence of such resampling steps repeated until all the indicators have been resampled. In the sampling implemented in the programs $l m \_$markers and $l m \_m u l t i p l e$ only inheritance vectors at marker loci are resampled. Thus each MCMC scan provides the next realization of $\mathrm{S}_{\mathrm{M}}$. Once a sequence of realizations of $S_{M}$ is obtained, equation (2) then provides a Monte Carlo estimate of the lod score as a function of the location of the trait locus.

At each MCMC iteration, our programs first randomly determine whether the scan is to be by locus (L-sampler) or by meiosis (M-sampler). Our new multiple meiosis (MM) sampler is a generalization of the M-sampler of Thompson and Heath [21] in which meioses are updated singly. For a subset $I$ of meioses, we write $S_{. j}=\left(S_{I j}, S_{-I j}\right)$, where $S_{I j}\left(S_{-I j}\right)$ denotes meiosis indicators in (not in) subset $I$ at locus $j$. Instead of one meiosis $i$, MM-sampler jointly resamples $\left\{S_{I j} ; j=1, \ldots, n\right\}$ successively over subsets $I$ of meioses conditionally on $\left\{S_{-I j} ; j=1, \ldots, n\right\}$ and the marker data $Y_{M}$. The factored HMM structure holds for this subset of meioses, so that the method of Fishelson and Geiger [25] can be applied for the calculations. 
We have defined several possible subsets $I$ : their benefits for MCMC mixing performance are considered further in the Discussion. Specifically, we consider subsets $I$ such as

1 random update: a random subset of the meioses, or

2 individual update: both maternal and paternal meioses for an individual, or

3 complete sib update: all the meioses from parents to children in a nuclear family, or

4 complete 3-generation update: all the meioses from grandparents to parents and from parents to children in a 3-generation family, which is defined as a nuclear family together with any grandparents present in the pedigree.

At each MCMC iteration of sampling inheritance indicators, a sampling type of a random update, individual update, sib update or 3-generation update is chosen according to probabilities $p_{n} p_{i}$, $p_{s}$ and $p_{3}$, where $p_{r}+p_{i}+p_{s}+p_{3}=1$. If a random update is chosen, then a random integer will first determine the size of this subset of meioses. For example, if the allowed maximum number of meioses in a subset is 8 , then a number will be selected from the integers from 1 to 8 each with probability $1 / 8$. This number of meioses will be selected randomly from the overall set of meioses and the indicators are updated according to its posterior distribution. Then a second integer from 1 to 8 will be randomly selected and a second subset of meioses randomly selected from the remaining meioses (excluding the ones previously selected), and so on until all the meiosis indicators are updated. If an individual update is chosen, the two meioses of each individual are updated jointly for each individual of the pedigree in a random order. Similarly, if a sib or 3-generation update is chosen, then the meioses included in these subsets are jointly updated, with the subsets being updated in random order. The subsets in a sib update are disjoint while the subsets in a 3-generation update can overlap since the grandparents in a 3-generation family can be parents or children in other 3-generation families.

\section{Restricted Multiple-Meiosis Updates}

The computational time is exponential in the number $k$ of meioses in subset $I$. When $k$ is too large, it could be computationally expensive or even impossible to do a complete sib or 3-generation update. In this case, a restricted sib or 3-generation update is possible.

For the restricted sib update, consider $I$ to be the set of meioses from parents to children in a nuclear family. Write $I=\left(I_{m}, I_{f}\right)$, where $I_{m}$ and $I_{f}$ are the maternal and paternal meioses in subset $I$. Given a current realization of meiosis indicators $s_{I .}=\left(s_{I 1}, \ldots, s_{I n}\right)$, for each locus $j=1, \ldots, n$, we define $X_{j}$ to be an indicator function of flipping paternal (or maternal) meiosis indicators or not in the next updating proposal. Specifically, we have

$$
X_{j}=\left\{\begin{array}{lll}
0 & \text { if } & S_{I_{m} j}=s_{I_{m} j}, S_{I_{f} j}=s_{I_{f} j} \\
1 & \text { if } & S_{I_{m} j}=1-s_{I_{m} j}, S_{I_{f} j}=s_{I_{f} j} \\
2 & \text { if } & S_{I_{m} j}=s_{I_{m} j}, S_{I_{f} j}=1-s_{I_{f} j} \\
3 & \text { if } & S_{I_{m} j}=1-s_{I_{m} j}, S_{I_{f} j}=1-s_{I_{f} j}
\end{array}\right.
$$

Similarly, when the complete 3-generation update is impractical, a restricted 3-generation update can be applied. Consider $I$ to be the set of meioses in a three generational family of grandparents, parents and children. Let $s_{I}=\left(s_{I m}, s_{I f}, s_{I c}\right)$ be the current realization of meiosis indicators in subset $I$, where $s_{I m}$ and $s_{I f}$ are the maternal and paternal meiosis indicators from grandparents to parents, and $s_{I c}$ are meiosis indicators from parents to children. For $j=$ $1, \ldots, n$, we define $X_{j}$ to be the following

$$
X_{j}=\left\{\begin{array}{l}
0 \text { if } S_{I j}=s_{I j} \\
1 \text { if } S_{I_{m} j}=s_{I_{f} j}, S_{I_{f} j}=s_{I_{m} j}, S_{I_{c} j}=1-s_{I_{c} j}
\end{array}\right.
$$

That is, there are two restricted choices to update meiosis indicators in I: (1) the same as current realization (2) swapping maternal and paternal meiosis indicators for meioses from grandparents to parents and flipping all the meiosis indicators for meioses from parents to children.

We show in the Appendix that $\left\{X_{j}\right\}_{j=1, \ldots, n}$ is a Markov chain for the case of restricted sib update. An analogous result holds for the case of the restricted 3-generation update. Hence, given data $Y_{. j}$ and fixed $S_{-I j}$ for $j=1, \ldots, n$, we retain the HMM structure with the $\left\{X_{j}\right\}_{j=1, \ldots, n}$ replacing the much larger space of $S_{I j}$. Since each $X_{j}$ takes only 2 or 4 values, it is possible to jointly sample $X=$ $\left(X_{1}, \ldots, X_{n}\right)$ for all the marker loci in time of order $n$ instead of the previous $n k 2^{k}$. The total number of choices over all $n$ loci is then $4^{n}$ or $2^{n}$ at each resampling step, providing a wide space of potential updated meiosis indicators. Updates similar to our restricted updates were proposed by Thomas et al. [26], for a single marker locus. However, they did not demonstrate how all the loci can be updated jointly.

\section{Haplotype Sampler: Combination of Exact and Approximate Calculations}

Conditional on the complete haplotypes at marker and trait loci of individuals who divide the pedigree into subsets of individuals, data on the different pedigree subsets are independent. The conditional independence of pedigree segments given the haplotypes of individuals who divide the pedigree is the basis of the Elston-Stewart algorithm [31] for likelihood computations on pedigrees. However, here we have multiple marker loci, so our method of computation on each pedigree segment is based on the Lander-Green approach [19]. We augment the space of hidden variables with the haplotypes of such 'key' individuals. If a subset separated by a 'key' individual is small, exact computation is feasible. In genetic studies, data are more often available for extant individuals at the bottom of the pedigree, constraining the haplotypes of immediate ancestors. It is therefore practical and efficient to do exact calculation on small subpedigrees of current individuals, taking as a 'key' individual the ancestor who connects the subpedigree to the remainder of the pedigree.

The haplotypes used to augment the space of hidden variables include the trait locus in addition to the marker loci. Without loss of generality, assume the trait locus $T$ is between the $d$ th and $d+1$ st marker loci. Then, for each meiosis $i,\left\{S_{i j}\right\}_{j=1, \ldots, d, T, d+1, \ldots, n}$ is a Markov chain, and the trait data depend only on the trait model and the inheritance at the trait locus. That is, the HMM structure and all the computational and sampling methods of the previous sections follow exactly as before, with the trait locus now being included.

For example, in the pedigree segment shown in figure 1, let $Y_{1}=\left(Y_{1 T}, Y_{1 M}\right)$ and $Y_{2}=\left(Y_{2 T}, Y_{2 M}\right)$ be the observed marker and trait data of 'key' individuals $\mathrm{C}$ and $\mathrm{D}$, and $H_{1}$ and $H_{2}$ be the pairs 


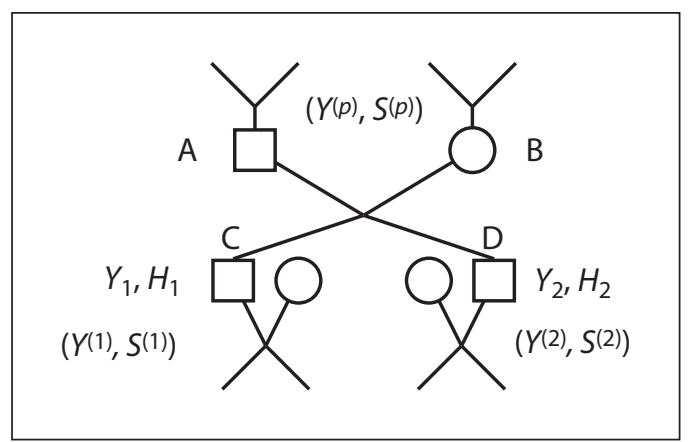

Fig. 1. An example of part of a complete pedigree, which includes ancestors and collateral relatives of individuals $\mathrm{A}$ and $\mathrm{B}$, and descendants and siblings of individuals $\mathrm{C}$ and $\mathrm{D}$. The two children $\mathrm{C}$ and $\mathrm{D}$ of the couple A and B are chosen as 'key' individuals, dividing the pedigree into three subpedigrees. For further details, see text.

of haplotypes of C and D who are offspring of the couple A and B. The total pedigree is then divided into three parts. The first descendant pedigree consists of C, C's descendants and all relatives of C's spouses. The second descendant pedigree is defined analogously for D. Finally, the ancestral pedigree consists of the remainder of the pedigree, including $\mathrm{C}$ and $\mathrm{D}$ themselves. Let $Y^{(p)}$, $S^{(p)}$ be data and meiosis indicators involved in the ancestral pedigree, $Y^{(1)} ; S^{(1)}\left(Y^{(2)}, S^{(2)}\right)$ be data and meiosis indicators in the 1 st (2nd) descendant pedigree. Note that, since the key individuals themselves are in both descendant and ancestral pedigree segments, $Y_{1}$ is included in both $Y^{(1)}$ and $Y^{(p)}$, and $Y_{2}$ is included in both $Y^{(2)}$ and $Y^{(p)}$. Then the probability distribution of the observed data $Y$ can be written as

$$
\begin{aligned}
P_{\gamma}(Y)= & \sum_{H_{1}, H_{2}} P_{\gamma}\left(Y^{(1)}, Y^{(2)} \mid Y_{1}, Y_{2}, H_{1}, H_{2}\right) P_{\gamma}\left(Y^{(p)}, H_{1}, H_{2}\right) \\
= & \sum_{S^{(p)}} \sum_{H_{1}, H_{2}} P_{\gamma}\left(Y^{(1)} \mid Y_{1}, H_{1}\right) P_{\gamma}\left(Y^{(2)} \mid Y_{2}, H_{2}\right) P_{\gamma}\left(Y^{(p)}, S^{(p)}\right) \\
& P\left(H_{1}, H_{2} \mid Y^{(p)}, S^{(p)}\right)
\end{aligned}
$$

Notice that in equation (3) the first term $P_{\gamma}\left(Y^{(1)} \mid Y_{1}, H_{1}\right)$ and second one $P_{\gamma}\left(Y^{(2)} \mid Y_{2}, H_{2}\right)$ are the same type, which only involves data in descendant pedigrees. The term $P_{\gamma}\left(Y^{(p)}, S^{(p)}\right)$ considers ancestral pedigree only. The last term $P\left(H_{1}, \mathrm{H}_{2} \mid Y^{(p)}, S^{(p)}\right)$ is a connection between descendant and ancestral pedigrees. We analyze these terms and explain in detail how to calculate them in the following four paragraphs.

First, consider the term $P_{\gamma}\left(Y^{(1)} \mid Y_{1}, H_{1}\right)$, or equivalently $P_{\gamma}\left(Y^{(2)} \mid Y_{2}, H_{2}\right)$. This part is independent of $S^{(p)}$, the meiosis indicators in the ancestral pedigree. Now

$$
P_{\gamma}\left(Y^{(1)} \mid Y_{1}, H_{1}\right)=\frac{P_{\gamma}\left(Y^{(1)}\right)}{P\left(H_{1}\right) P\left(Y_{1} \mid H_{1}\right)} P_{\gamma}\left(H_{1} \mid Y^{(1)}\right) .
$$

The probability for the first descendant part $P_{\gamma}\left(Y^{(1)}\right)$ can either be calculated exactly or, if it is too large, estimated by MCMC. For a fixed genetic map, $P_{\gamma}\left(Y^{(1)}\right)$ is calculated only once summing over all possible values of $H_{1}$ and $H_{2}$. The prior probability, $P\left(H_{1}\right)$, of the ordered haplotype pair $H_{1}$, is assumed to be the product of the allele frequencies at each marker/trait locus. The multilocus penetrance probability $P\left(Y_{1} \mid H_{1}\right)$ is a product over the marker and trait loci, 1 or 0 for genotypic data, or a more general penetrance for discrete or quantitative trait data. When $P\left(Y_{1} \mid H_{1}\right)=0$, $P_{\gamma}\left(Y^{(1)} \mid Y_{1}, H_{1}\right)$ is defined to be 0 . The haplotype pair $h_{1}$ can be sampled according to the posterior distribution $P_{\gamma}\left(H_{1} \mid Y^{(1)}\right)$. When the number of possible haplotypes is not large, the full distribution $P_{\gamma}\left(H_{1} \mid Y^{(1)}\right)$ can be calculated exactly. However, when the exact calculation is infeasible, we have

$$
\begin{aligned}
& P_{\gamma}\left(H_{1} \mid Y^{(1)}\right)=\sum_{S^{(1)}} P_{\gamma}\left(S^{(1)} \mid Y^{(1)}\right) P\left(H_{1} \mid Y^{(1)}, S^{(1)}\right) \\
= & \sum_{S^{(1)}} P_{\gamma}\left(S^{(1)} \mid Y^{(1)}\right)\left[\frac{P\left(H_{1 T}, Y_{\cdot T}^{(1)} \mid S_{\cdot T}^{(1)}\right)}{P\left(Y_{\cdot T}^{(1)} \mid S_{\cdot T}^{(1)}\right)} \prod_{j=1}^{n} \frac{P\left(H_{1 j}, Y_{\cdot j}^{(1)} \mid S_{\cdot j}^{(1)}\right)}{P\left(Y_{\cdot j}^{(1)} \mid S_{\cdot j}^{(1)}\right)}\right]
\end{aligned}
$$

Equation (4) indicates that we can first sample $s^{(1)}$ conditional on $Y^{(1)}$. We then sample $h_{1}$, locus by locus, conditional on $Y^{(1)}$ and a realization of $S^{(1)}$.

Second, consider the term $P_{\gamma}\left(Y^{(p)}, S^{(p)}\right)$ in equation (3). We use MCMC to sample $s^{(p)}$ conditional on data $Y^{(p)}$ using the new MMsampler to improve mixing. Now

$$
P_{\gamma}\left(Y^{(p)}, S^{(p)}\right)=P\left(S_{M}^{(p)} \mid Y_{M}^{(p)}\right) P_{\gamma}\left(S_{T}^{(p)} \mid S_{M}^{(p)}\right) P\left(Y_{T}^{(p)} \mid S_{T}^{(p)}\right) P\left(Y_{M}^{(p)}\right) .
$$

Thus we may sample $s^{(p)}=\left(s_{M}^{(p)}, s_{T}^{(p)}\right)$ by first sampling $s_{M}^{(p)}$ from $P_{\gamma}\left(S_{M}^{(p)} \mid Y_{M}^{(p)}\right)$ and then $s_{T}^{(p)}$ from $P_{\gamma}\left(S_{T}^{(p)} \mid s_{M}^{(p)}\right)$. The trait-locus penetrance probability on the ancestral pedigree, $\left.P\left(Y_{T}^{(p)}\right\rfloor_{T}^{(p)}\right)$, can be easily calculated for a given $s_{T}^{(p)}$. The last term $P\left(Y_{M}^{(p)}\right)$ is free of parameter $\gamma$ and the values of $S^{(p)}, H_{1}$ and $H_{2}$. Thus to obtain a lod score, it is not necessary to calculate this term.

Finally, consider the term $P\left(H_{1}, H_{2} \mid Y^{(p)}, S^{(p)}\right)$ in equation (3). Similarly to equation (4), this probability can be easily calculated locus by locus

$$
\frac{P\left(H_{1 T}, H_{2 T}, Y_{\cdot T}^{(p)} \mid S_{\cdot T}^{(p)}\right)}{P\left(Y_{\cdot T}^{(p)} \mid S_{\cdot T}^{(p)}\right)} \prod_{j=1}^{n} \frac{P\left(H_{1 j}, H_{2 j}, Y_{\cdot j}^{(p)} \mid S_{\cdot j}^{(p)}\right)}{P\left(Y_{\cdot j}^{(p)} \mid S_{\cdot j}^{(p)}\right)} .
$$

To summarize, we estimate the likelihood of equation (3) by first sampling $h_{1} \sim P_{\gamma}\left(H_{1} \mid Y^{(1)}\right)$ and $h_{2} \sim P_{\gamma}\left(H_{2} \mid Y^{(2)}\right)$; then sampling $s_{M}^{(p)} \sim P\left(S_{M}^{(p)} \mid Y_{M}^{(p)}\right)$, and $s_{T}^{(p)} \sim P_{\gamma}\left(S_{T}^{(p)} \mid \begin{array}{c}(p) \\ s_{M}\end{array}\right)$; finally calculating

$$
\frac{P\left(Y_{T}^{(p)} \mid S_{T}^{(p)}\right) P\left(H_{1}=h_{1}, H_{2}=h_{2} \mid Y^{(p)}, s^{(p)}\right) P_{\gamma}\left(Y^{(1)}\right) P_{\gamma}\left(Y^{(2)}\right)}{P\left(Y_{1} \mid H_{1}=h_{1}\right) P\left(H_{1}=h_{1}\right) P\left(Y_{2} \mid H_{2}=h_{2}\right) P\left(H_{2}=h_{2}\right)} .
$$

The average of this quantity over MCMC realizations provides an estimate of $P_{\gamma}\left(Y \mid Y_{M}^{(p)}\right)$, which is proportional to the likelihood

$$
\mathrm{L}(\gamma)=P_{\gamma}(Y)=P_{\gamma}\left(Y \mid Y_{M}^{(p)}\right) P\left(Y_{M}^{(p)}\right) .
$$

Of course, this method is not limited to the special case of two 'key' individuals who are siblings. One can choose any small number of individuals anywhere in the pedigree as 'key' individuals. However, different choices of 'key' individuals can make a difference in the efficiency of estimation. As a guideline, one 
Fig. 2. Ped52 pedigree used for simulation of data. The individuals in grey are not observed - both marker genotype and affectation status are missing. The white individuals are unaffected and the black ones are affected.

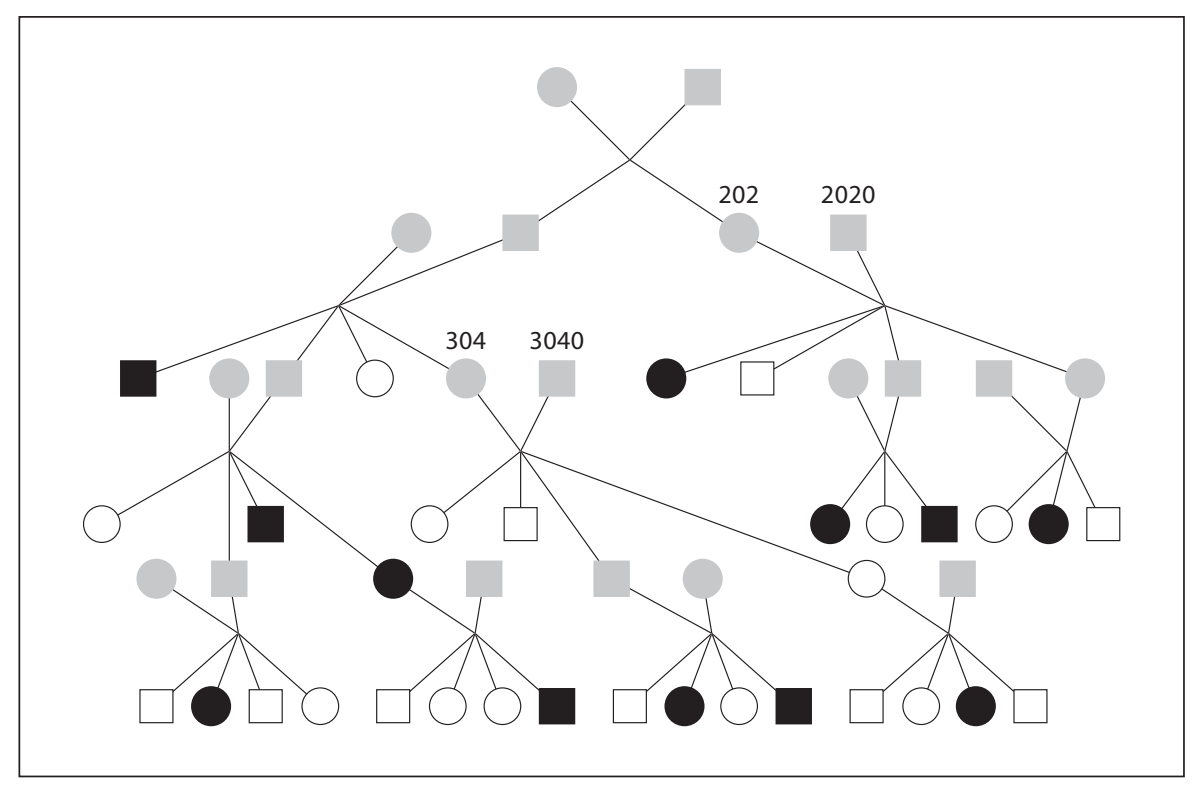

should choose individuals whose descendants have enough information to at least partially restrict the space of haplotypes of each key individual. Additionally, the procedure is much more effective if exact computation is used on the descendant pedigree segments. Thus, the number of each individual's descendants should be not large so that the computation of the exact haplotype distribution is infeasible.

The methods of this section have been implemented in the program $l m$ _haplotype. In this program, a subset of 'key' individuals must be predefined by users. Then the program $I m \_$haplotype will automatically divide the whole pedigree into parts and calculate each part exactly or approximately according to the size of each part and the maximum number of meiosis allowed for exact calculations. When Monte Carlo approximation is necessary, the MM-sampling of $l m \_$multiple, rather than the M-sampler of $l m$ markers, is applied. The final results are then combined as described above and estimates of lod scores are returned.

\section{Results}

\section{Set Up for Simulation and Analysis}

We illustrate our methods on the single pedigree, ped52, shown in figure 2 . The pedigree has 52 individuals in 5 generations: 12 individuals are founders, 32 are observed, 12 are affected, and 20 are unaffected. There are 80 meioses to be sampled. Data were simulated at 10 linked marker loci, labeled from 1 to 10 , at chromosomal positions $(0,10,20,28,29,31,40,50,60,61) \mathrm{cM}$. Each marker locus has 4 alleles, with allele frequencies $(0.4,0.3$, $0.2,0.1)$. The trait locus is simulated at position $30 \mathrm{cM}$. Note that the trait locus is in a region of tightly linked markers: markers 4, 5 and 6 and the trait locus present a particular challenge for MCMC methods. The trait locus has 2 alleles with frequencies $(0.5,0.5)$. An affectation status with penetrances $(0.95,0.6,0.05)$ is simulated. In the analysis, trait-locus genotypes are unobserved, but affectation status is available for each of the 32 observed individuals. The assumption of 'known' phenotypes of unaffected individuals results in a stronger linkage signal compared to many real data analyses, where often only the more clearly defined 'affected' phenotype is specified. However, this fact has no (in lm_multiple) or little (in lm_haplotype) effect on the mixing performance of MCMC.

All the programs were run on a Dell Precision 360 workstation with Pentium $4(3 \mathrm{GHz})$ processor, $2 \mathrm{~GB}$ memory and Red Hat Enterprise Linux 4 WS system. In all three MCMC programs, we use $3 \mathrm{~K}(3,000)$ sequential imputation realizations to obtain the initial realization of meiosis indicators $S_{M}$. The value chosen is the realization that gives the highest value of $P\left(Y_{T} \mid S_{M}\right)$. In lm_markers and $l m \_$multiple, the probabilities for $\mathrm{L}$ and $\mathrm{M}$ sampling are 0.5 and 0.5 respectively. Sampling is by scan in both lm_markers and $l m \_$multiple. When $l m \_$multiple selects an M-sample scan, the probabilities for individual, sib, 3generation and random updates are $0.3,0.3,0.2$ and 0.2 respectively. In the random update, the number $k$ of meioses jointly updated is uniformly distributed from 1 to 8 . In the sib and 3-generation updates, the maximum allowed number of meioses for complete updating is 8 . That is, when the number of involved meioses in these updates is 


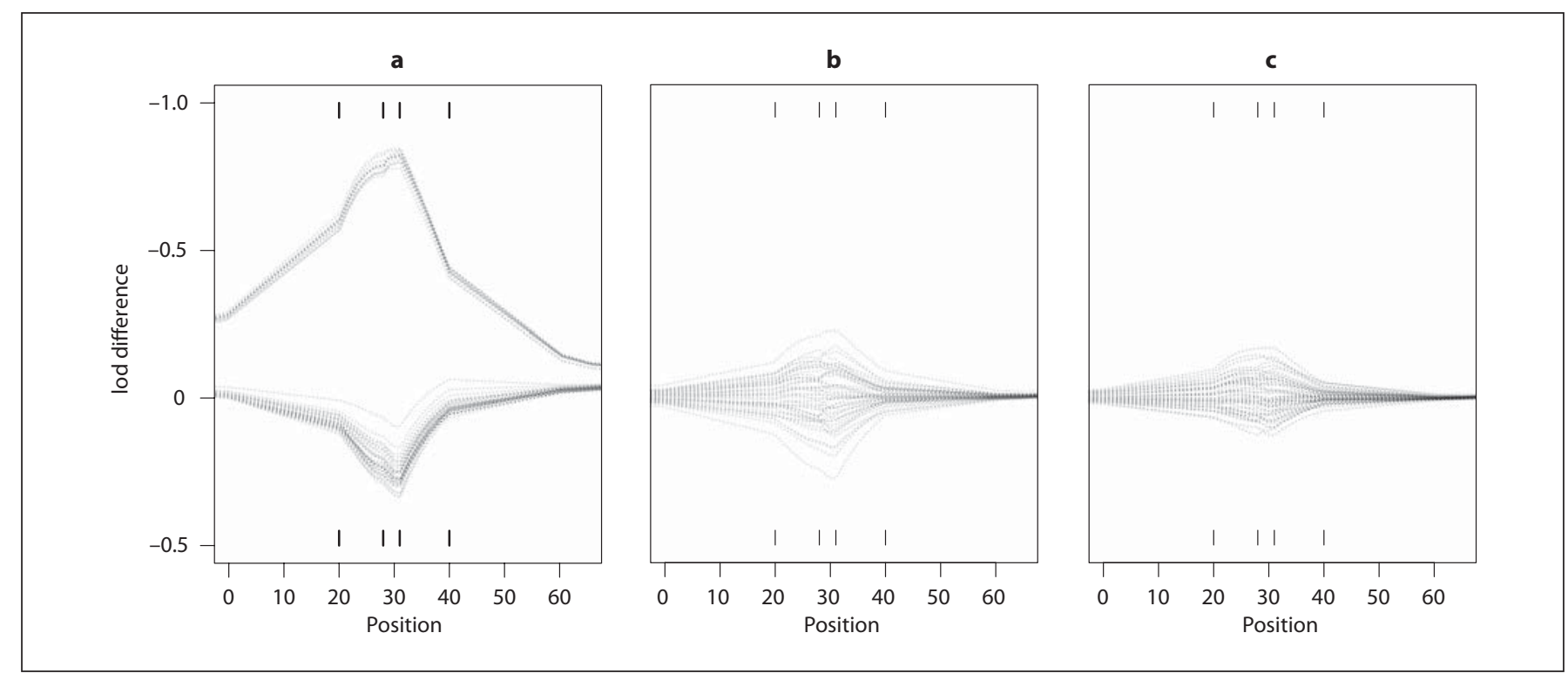

Fig. 3. Differences between exact lod scores and MCMC estimates for ped52 with 4 markers at positions 20, 28, 31 and $40 \mathrm{cM}$. The exact lod scores are calculated using VITESSE. From left to right, the three MCMC programs used in estimation are $l m \_$markers (a), lm_multiple (b), lm_haplotype (c). For each program, the number of runs is 50 . For each run, the number of scans is $30 \mathrm{~K}$.

greater than 8 , restricted updates are applied. For $l m \_h a p$ lotype, whenever MCMC sampling is necessary on either ancestral or descendant parts of the pedigree, $l m \_$multiple is used with the same parameter values as above.

To choose the 'optimal' key individuals for $l m \_h a p l o-$ type, 50 short $l m \_$multiple runs (each of length $5 \mathrm{~K} \mathrm{MCMC}$ scans) are performed on some subpedigrees of ped52. For example, individuals 202, 2,020 and their descendants would be one such subpedigree (fig. 2). Another example subpedigree would be individuals 304, 3040 and their descendants. The idea is that special attention should be paid to the subpedigrees that give significant contribution to the overall lod scores but have large variation among independent runs. Using this method, we find that in this example, the subpedigree that might be the source of large variation is the one consisting of individuals 202, 2020 and their descendants. There are 14 individuals and 20 meioses in this subpedigree. For $l m \_h a p$ lotype, we then choose individual 202 as the key individual and sample her haplotype, conditional the data on this subpedigree. The computation time for this preliminary analysis is not included in table 2 .

\section{Ped52 with 4 Markers}

Our aim is to obtain accurate MCMC lod score estimates for large pedigrees with multiple markers. How- ever, on ped52 we first consider markers 3, 4, 6, 7 only (ped52-4), in order to make possible a comparison with exact computations.

The exact lod scores are calculated using VITESSE [28] and is shown in figure 5 (dashed line). The differences between exact lod scores and estimated ones from 50 runs using the three MCMC programs $l m \_$markers, lm_multiple and Im_haplotype (30K MCMC scans for each run) are shown in figure 3. For $l m \_$markers (fig. 3a), there are two clear separate clusters of estimates, indicating poor MCMC mixing. Therefore, the estimated lod scores are not accurate and reliable in this case. To determine whether a longer run can overcome this mixing problem, we choose 10 runs from the upper cluster and 10 runs from the lower one and extend the number of MCMC scans from $30 \mathrm{~K}$ to $1 \mathrm{M}(1,000,000)$ for each run. All the 20 runs are still in their original cluster after such a long run, although the between-run variation of lod scores within each cluster does decrease compared to the lod score estimates based on 30K MCMC scans (results not shown). This indicates that the $l m \_$markers sampling procedure has negligible chance of moving between subsets of $S_{M}$ that provide lod scores in different clusters in this example.

Figure $3 \mathrm{~b}$ shows that $l m \_$multiple successfully overcomes the mixing difficulties and obtains lod scores 
Table 1. Comparison of programs: lod score accuracy and variation

\begin{tabular}{|c|c|c|c|c|c|c|c|c|}
\hline \multirow[t]{2}{*}{ Pedigree } & \multirow[t]{2}{*}{ Position (lod) } & \multirow{2}{*}{$\begin{array}{l}\text { \# of MCMC } \\
\text { scans }\end{array}$} & \multicolumn{3}{|c|}{ Discrepancy } & \multicolumn{3}{|l|}{ Range } \\
\hline & & & mrk & mul & hap & mrk & mul & hap \\
\hline \multirow[t]{9}{*}{ ped52-4 } & Marker-3 (0.568) & $10 \mathrm{~K}$ & 0.217 & 0.073 & 0.050 & 0.827 & 0.342 & 0.248 \\
\hline & & $30 \mathrm{~K}$ & 0.249 & 0.044 & 0.032 & 0.754 & 0.245 & 0.163 \\
\hline & & $1 \mathrm{M}$ & 0.156 & 0.008 & 0.008 & 0.675 & 0.042 & 0.034 \\
\hline & Marker-6 (0.965) & $10 \mathrm{~K}$ & 0.405 & 0.143 & 0.095 & 1.231 & 0.594 & 0.487 \\
\hline & & $30 \mathrm{~K}$ & 0.439 & 0.082 & 0.057 & 1.195 & 0.504 & 0.299 \\
\hline & & $1 \mathrm{M}$ & 0.335 & 0.014 & 0.012 & 1.091 & 0.081 & 0.071 \\
\hline & Marker-7 (0.776) & $10 \mathrm{~K}$ & 0.140 & 0.040 & 0.028 & 0.554 & 0.176 & 0.131 \\
\hline & & $30 \mathrm{~K}$ & 0.162 & 0.023 & 0.017 & 0.510 & 0.148 & 0.095 \\
\hline & & $1 \mathrm{M}$ & 0.093 & 0.004 & 0.004 & 0.472 & 0.023 & 0.020 \\
\hline \multirow[t]{6}{*}{ ped14-10 } & Marker-3 (0.224) & $10 \mathrm{~K}$ & 0.012 & 0.006 & 0.004 & 0.056 & 0.032 & 0.024 \\
\hline & & $30 \mathrm{~K}$ & 0.007 & 0.004 & 0.003 & 0.038 & 0.021 & 0.014 \\
\hline & Marker-6 (0.646) & $10 \mathrm{~K}$ & 0.027 & 0.012 & 0.007 & 0.135 & 0.065 & 0.041 \\
\hline & & $30 \mathrm{~K}$ & 0.020 & 0.008 & 0.006 & 0.122 & 0.042 & 0.031 \\
\hline & Marker-7 (0.636) & $10 \mathrm{~K}$ & 0.018 & 0.010 & 0.006 & 0.108 & 0.055 & 0.039 \\
\hline & & $30 \mathrm{~K}$ & 0.016 & 0.006 & 0.004 & 0.120 & 0.034 & 0.026 \\
\hline
\end{tabular}

The number $10 \mathrm{~K}$ means 10,000 and $1 \mathrm{M}$ means 1 million. The numbers included in parentheses in the position column are exact lod scores calculated by VITESSE or MERLIN at these positions. mrk, mul and hap represent lm-markers, lm-multiple and lm-happlotype respectively.

varying around true lod-score curve. Figure $3 \mathrm{c}$ shows that $l m \_$haplotype not only overcomes the mixing difficulties of $l m$ markers, but also has smaller variation compared to lm_multiple. In the worst scenarios of bad choices of key individuals, Im_haplotype performs at least as well as $l m \_$multiple (results not shown).

To see how the number of MCMC scans affects the final estimates, we also did 50 short runs ( $10 \mathrm{~K}$ scans per run), 50 long runs ( $30 \mathrm{~K}$ scans per run) and 50 extremely long runs (1M scans per run). For a given position, we consider two measures of accuracy and precision of the estimated lod score: the discrepancy which is the mean (over runs) of the absolute difference from the truth, and the range which is the difference between maximum and minimum over runs. For each program, these 2 statistics are calculated at three positions: marker-3, marker-6, and marker-7. The results are shown in table 1.

From table 1 we see that for ped52 with 4 markers, both $l m \_$haplotype and $l m \_$multiple have much smaller discrepancy than $l m \_$markers. For example, for the long runs at marker-6, Im_haplotype, $I m$ _multiple and $I m_{-}$ markers have discrepancies $0.057,0.082,0.439$ respectively, which are 6,8 and $45 \%$ of the true lod score 0.965 at this position. With extremely long runs, the discrepan-
Table 2. Comparison of programs: computation time in seconds

\begin{tabular}{|c|c|c|c|c|c|}
\hline \multirow[t]{2}{*}{ Pedigree } & \multirow[t]{2}{*}{ Exact } & \multirow{2}{*}{$\begin{array}{l}\text { \# of MCMC } \\
\text { scans }\end{array}$} & \multicolumn{3}{|c|}{ MCMC programs } \\
\hline & & & mrk & mul & hap \\
\hline \multirow[t]{3}{*}{ ped52-4 } & VITESSE & $10 \mathrm{~K}$ & 49 & 137 & 145 \\
\hline & $(19,000)$ & $30 \mathrm{~K}$ & 128 & 404 & 420 \\
\hline & & $1 \mathrm{M}$ & 4,112 & 12,467 & 12,530 \\
\hline \multirow[t]{2}{*}{ ped14-10 } & MERLIN & $10 \mathrm{~K}$ & 17 & 34 & 38 \\
\hline & $(2)$ & $30 \mathrm{~K}$ & 46 & 98 & 106 \\
\hline \multirow[t]{2}{*}{ ped52-10 } & - & $10 \mathrm{~K}$ & 86 & 317 & 332 \\
\hline & & $30 \mathrm{~K}$ & 223 & 931 & 945 \\
\hline
\end{tabular}

Each MCMC computation time is an average over the corresponding set of 50 runs of table 1 . The computation time of VITESSE and MERLIN are shown in parentheses in the exact column.

cies for these three programs decreased to $0.012,0.014$, 0.335 respectively. However, the discrepancy for $l m_{-}$ markers (0.335) is still too large to be reliable. This shows that $l m \_$markers can not overcome the mixing difficulties even with extremely long runs (1M scans). Moreover, 


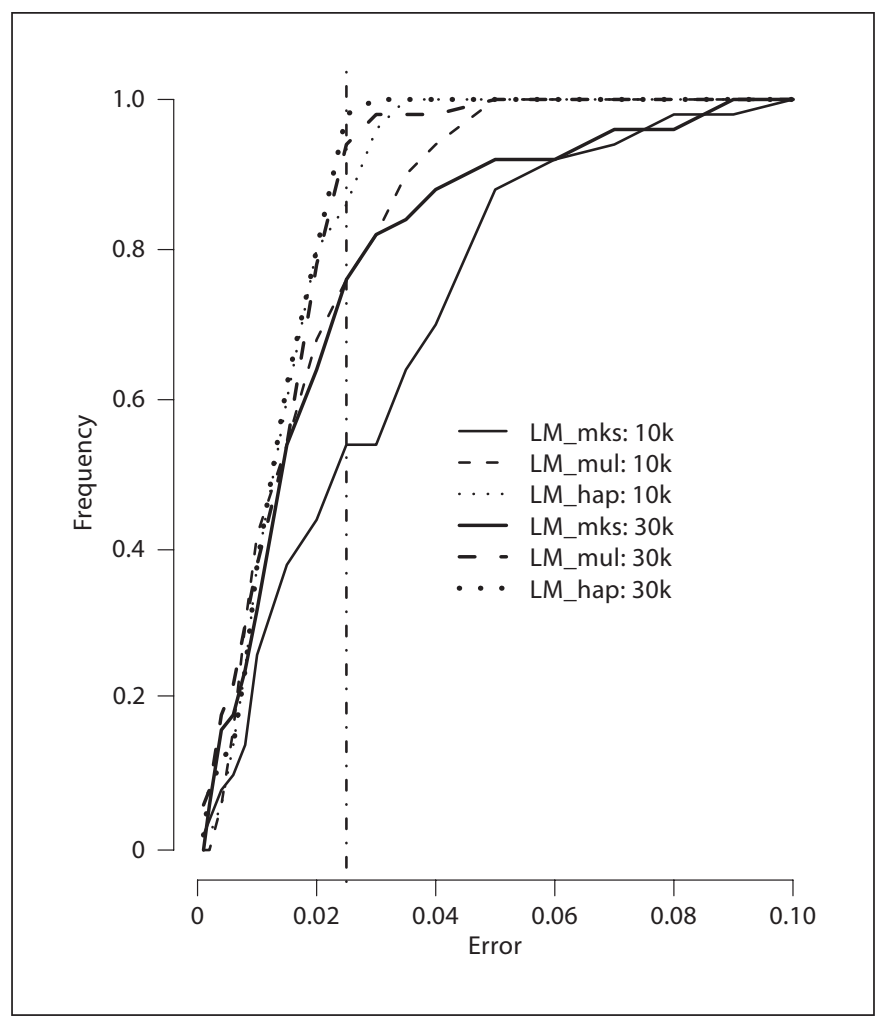

Fig. 4. Empirical CDF of errors of lod scores at true trait locus using $l m \_$markers, $l m \_$multiple and $l m \_$haplotype.

lm_haplotype and lm_multiple also have smaller range than $l m \_$markers in most cases.

For a given number of MCMC scans, the computation time for $l m_{-}$haplotype is about the same as for $l m_{-} m u l-$ tiple and about three times the time for $l m_{-}$markers (table 2). That is, a short run (10K scans) of $l m \_m u l t i p l e$ or lm_haplotype uses about the same computation time as a long run (30K scans) of $l m \_$markers. By comparison, note that exact computation using VITESSE [28] takes much longer time to compute the lod scores at the same points as the MCMC programs estimate them (table 2). On this pedigree, four 4-allele markers is the limit of computation feasibility for VITESSE, showing the importance of having accurate and reliable MCMC methods.

For the three MCMC programs, a fair comparison with respect to time is thus of the long run results using lm_markers with the short run results using $l m \_m u l t i p l e$ and lm_haplotype. Table 1 shows that even short runs (10K scans) of lm_haplotypes and lm_multiple perform much better than long runs (30K scans) of lm_markers, with both smaller discrepancy and smaller range over runs. Notice that, in this example, more MCMC scans of Im_markers do not necessarily increase accuracy or decrease variability because of poor mixing of the Markov chain. However, increasing the number of MCMC scans for $l m \_$haplotype or for $l m \_m u l t i p l e$ does decrease both the discrepancy and the range.

\section{Ped14 with 10 Markers}

To use all 10 markers and to compare the performance of these with exact calculation, we can use only a small part (ped14-10) of the pedigree. We consider the right part of ped52 consisting of founders 202, 2,020 and their descendants: 14 individuals in total (see fig. 2). MERLIN [29] can compute exact lod scores on this subpedigree. For each of the three MCMC programs, the discrepancy and range at marker- 3 , at marker- 6 , and at marker- 7 are shown in table 1. Comparing the long run results from lm_markers with short run results from $l m \_$multiple and lm_haplotype, we find that the latter two programs always have smaller discrepancy and less variation (table 1). In fact, in this example, lm_markers requires about $50 \%$ more computation time for $30 \mathrm{~K}$ MCMC scans than do the other two programs for $10 \mathrm{~K}$ scans (table 2).

A further comparison of the accuracy of MCMC estimates as compared to exact results from MERLIN is shown in figure 4 . For all three MCMC programs, and for both short and long runs, this figure shows the empirical CDF (over 50 runs) of absolute error in lod score at the true trait locus (position $30 \mathrm{cM}$, at the midpoint between marker-5 and marker-6). For example, consider the number of runs (out of 50) with this error less than or equal to 0.025 , which is $4.8 \%$ of the true lod score of 0.518 . For short and long runs, respectively, this number is about 27 and 38 for $l m$ _markers, 38 and 47 for lm_multiple, and 43 and 49 for $l m \_$haplotypes. In this example, lm_markers does not have the obvious mixing problem that it had on the full pedigree; there are no distinct clusters of lod score estimates among the 50 short or long runs. However, $l m_{-}$ multiple and $l m_{-}$haplotype still perform better, providing more accurate estimates with less variability in shorter time.

\section{Ped52 with 10 Markers}

We estimate the lod score for the complete ped52 pedigree with all 10 markers (ped52-10) using these three MCMC programs. As before, we obtain 50 independent long runs (30K MCMC scans) for each program. Now, no comparison with exact results is possible, so the discrepancy measure is not obtainable. The lod score ranges at marker-6, for lm_markers, Im_multiple and $l m \_$haplo- 
type, are $0.466,0.059$, and 0.032 , respectively. As before we get the smallest range for $m$ _haplotype and the largest range for $l m$ markers. Comparing with the corresponding results for ped52-4 (table 1), we see that there is much less between-run variation in the lod score using all 10 markers.

To check how the between-run variation in the lod score is affected by markers included in the analysis, we estimate the lod score for the complete ped52 pedigree with 5 markers of 3, 4, 5, 6, 7 (ped52-5). Exact results are not possible here. Using the results from 50 independent long runs (30K MCMC scans) for each program (results not shown), the lod score ranges at marker-6, for $l m_{-}$ markers, lm_multiple and lm_haplotype, are 0.307, 0.031, and 0.021 , respecitvely. The between run variations are not only less than the ones for ped 52 using 4 markers, but also less than the ones for ped52 using all 10 markers. This shows that marker-5 plays an important role in estimating lod scores at marker-6.

Finally, the lod-score curve is estimated for ped52-10 using our best program Im_haplotype with $1 \mathrm{M}$ scans (fig. 5). For comparison, figure 5 shows also the exact lod scores for ped52-4 and the estimated lod scores for ped525. The estimated lod score curve for ped52-5 is the average over the 50 long runs using $l m \_$haplotype. It takes about 2 hours to get the MCMC-based ped52-10 lod score curve. Based on the consistent performance of $l m \_$haplotype for ped52-4 and ped14-10, we are confident that this lod-score curve is reliable. The maximum lod score 1.972 is obtained at marker- 6 for ped52-10, which is more than the maximum lod score of 1.771 from ped52-5 and about twice of the maximum lod score from ped52-4. This shows that the increase in maximum lod score is mainly, but not entirely, due to the marker data at marker 5. For extended pedigrees with many missing data, we thus see that joint use of data at multiple markers increases the power to detect linkage.

\section{Discussion}

We have developed new MCMC methods for accurate estimation of multilocus likelihoods using pedigree data. MCMC methods make linkage analysis feasible for large pedigree data when exact computational methods cannot be applied (ped52-10). Even in the case that exact computation is feasible, MCMC methods can give reasonable results using much less computational time (ped52-4).

Compared to the $l m$ _markers method, our new MCMC methods improve the accuracy of lod score estimates and

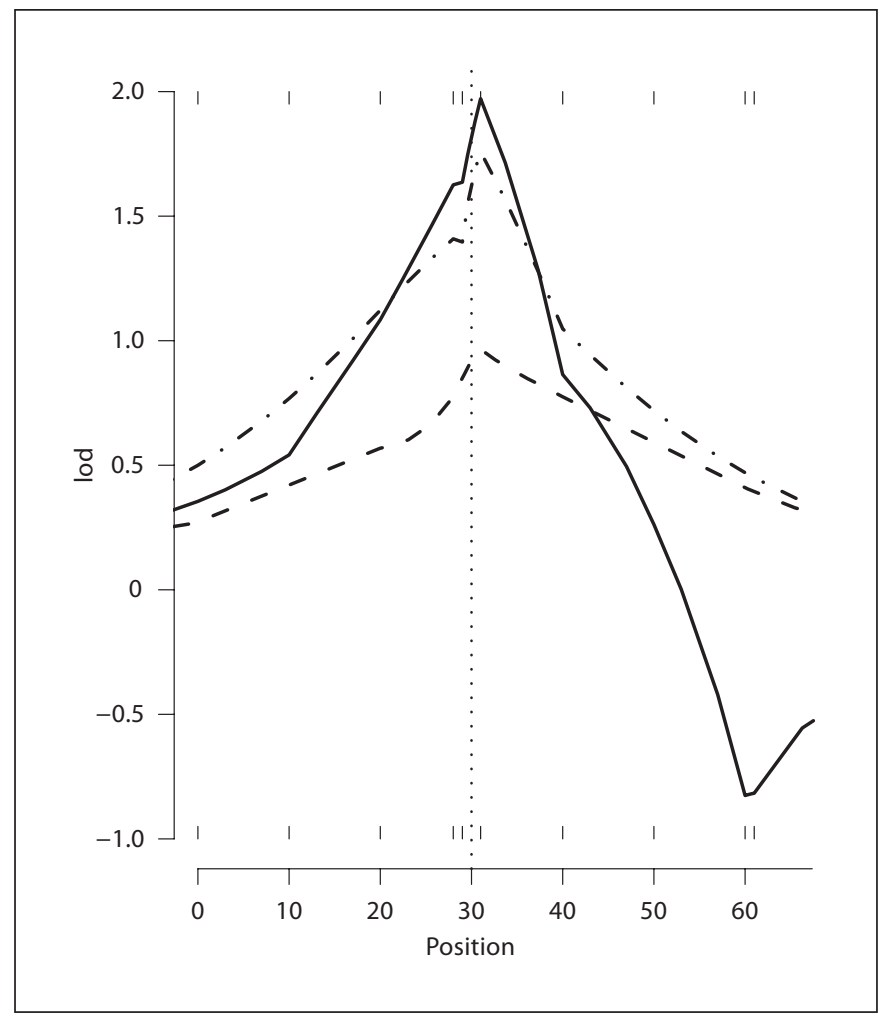

Fig. 5. The solid line is the estimated lod score curve for ped52-10 using $l m$ _haplotype with $1 \mathrm{M}$ scans. The dot-dashed line is the estimated lod score curve for ped52-5 at positions 20, 28, 29, 31, 40 $\mathrm{cM}$ and dashed line is the exact lod score curve for ped52-4 at positions 20,28, 31, $40 \mathrm{cM}$. The vertical dotted line at $30 \mathrm{cM}$ represents the position of simulated trait locus.

decrease the variation of lod score estimates between runs. The procedure $l m \_$multiple outperforms $l m \_$markers by jointly considering multiple meioses, and $l m \_h a p-$ lotypes outperforms $l m \_$multiple by additionally considering haplotypes of some individuals.

By updating jointly over loci, a single meiosis sampler (M-sampler) avoids problems of poor mixing due to tight linkage [21], but inheritances in different meioses are jointly constrained by data. Further, although the L-sampler and hence also the LM-sampler are irreducible, the M-sampler alone can never be guaranteed irreducible. Clearly, the random, individual, complete sib and 3-generation updates improve mixing; they may sometimes even ensure irreducibility of the sampler. Often the joint updating of meioses within nuclear families may be sufficient to improve mixing, whereas the random update could (in principle) choose meioses whose inheritances are independent given the data. In this extreme case, the 
MM-update process is equivalent to M-sampler updates of this block of meioses but requires longer computation time. However, as shown by the example of Sobel and Lange [27], two meioses well separated in the pedigree may be jointly constrained by the data on descendants, causing reducibility of single-meiosis updates. Thus it seems advisable to always give positive probability to the updating of randomly chosen subsets, and not restrict the process only to local sib or even 3-generation updates.

In $l m \_$multiple, the probabilities for random, individual, sib and 3-generation updates are pre-defined. Higher probability of complete sib and 3-generation updates may provide better estimates of lod scores because the meiosis indicators are jointly updated according to full conditional distribution. However, it remains to be investigated whether the gains outweigh the additional computational burden. Similarly, for lm_haplotype, exact calculations can be done for small parts of the pedigree. However, exact calculation is computationally intensive and the choice of how much exact computation to do is always a compromise between accuracy and time.

In lm_haplotype, the choice of key individuals has effects on both the performance of MCMC and the computation time required. In principle, any individual can be a key individual. However, we would like to do as much exact calculation as possible since this computation can be done once only, and the more exact computation is done the smaller the Monte Carlo variation in the lod score estimate. Thus we prefer to choose a key individual that has a deeper descendant pedigree provided exact computation can still be done on this sub-pedigree. In the current analysis, we use short runs in partial pedigrees to find the underlying problematic part of the pedigree. Although this worked well here, more theoretical exploration is needed to support generalization. An automated procedure to choose optimal key individuals is also desirable.

All the three simulation studies are based on data from simple structured pedigree ped52. For complicated pedigree with loops, $l m \_$markers and $l m \_$multiple can be used without any modification, although loops may result in poor mixing in some cases. To use lm_haplotype, a little bit more modification in likelihood equation in equation

(3) is needed, when some key individuals and their descendants actually form a loop. More investigation is needed on the effect of loops on MCMC mixing performance.

The examples of this paper have used microsatellite type markers, but increasingly data are available for large numbers of dense SNP markers. For a given number of
MCMC scans, both $l m$ markers and lm_multiple have computation time linear in the number of marker loci $n$. The program $l m \_$haplotype is also linear in $n$ in the case that the haplotypes of key individuals are sampled conditional on realizations of meiosis indicators in sub-pedigrees. Wijsman et al. [24] have shown that $l m \_$markers can obtain accurate lod score estimates using dense simulated SNP marker data. In an analysis of the real SNP data of GAW15 [32], Im_multiple shows better performance than $l m \_$markers. Therefore, we are optimistic on the general performance of $l m \_$multiple using dense SNP data. More work is needed to evaluate the performance of Im_haplotype on SNP data.

Although it is always very challenging to deal with real, large pedigrees with multiple tightly linked marker loci and a lot of missing data, our new methods are promising in improving the performance over previous MCMC methods.

\section{Acknowledgments}

This work is supported by NIH grant GM-46255. The authors are very grateful to a referee whose many detailed and constructive comments have much improved the paper.

\section{Appendix 1}

1. Proof of Markov Property in Restricted Sib Update

For $j=1, \ldots, n$, let $s_{I j}^{*}=\left(\mathrm{s}_{I_{m j}}^{*}, \mathrm{~s}_{I_{f}}^{*}\right)$ be one of the four possible choices based on current realization $s_{I j}$ (flip or not for paternal or maternal meiosis indicators). Then for $k_{j} \in\{0,1,2,3\}$,

$$
\begin{aligned}
P(X) & =P\left(X_{1}=k_{1}, \ldots, X_{n}=k_{n}\right) \\
& \propto P\left(S_{I 1}=s_{I 1}^{*}, \ldots, S_{I n}=s_{I n}^{*}\right) \\
& =P\left(S_{I 1}=s_{I 1}^{*}\right) \prod_{j=2}^{n} P\left(S_{I j}=s_{I j}^{*} \mid S_{I j-1}=s_{I j-1}^{*}\right) \\
& =g\left(k_{1}\right) \prod_{j=2}^{n} g\left(k_{j}, k_{j-1}\right),
\end{aligned}
$$

where $g(k)$ is a function of $k$. This indicates that $\left\{X_{j}\right\}_{j=1, \ldots, n}$ is a Markov chain. Thus, using the HMM algorithm of Baum and Petrie [33] and Rabiner [34], we are able to sample $P(X)$ jointly over $n$ marker loci using block Gibbs sampling method in time of order $O(n)$. 


\section{References}

1 Ott J: Analysis of Human Genetic Linkage, ed 3. Baltimore, MD, The Johns Hopkins University Press, 1999.

-2 Abecasis GR, Yashar BM, Zhao Y, Ghiasvand NM, Zareparsi S, Branham KEH, Reddick AC, Trager EH, Yoshida S, Bahling J, Filippova E, Elner S, Johnson MW, Vine AK, Sieving PA, Jacobson SG, Richards JE, Swaroop A: Age-related macular degeneration: a high-resolution genome scan for susceptibility loci in a population enriched for latestage disease. Am J Hum Genet 2004;74: 482-494.

-3 International Hapmap Consortium. A haplotype map of the human genome. Nature 2005;237:1299-1319.

-4 Maraganore DM, de Andrade M, Lesnick TG, Strain KJ, Farrer MJ, Rocca WA, Pant PVK, Frazer KA, Cox DR, Ballinger DG: High-resolution whole-genome association study of Parkinson disease. Am J Hum Genet 2005;77:685-693.

5 Myers RH: Considerations for genomewide association studies in Parkinson disease. Am J Hum Genet 2006;78:1081-1082.

-6 Clarimon J, Scholz S, Fung HC, Hardy J, Eerola J, Hellstrm O, Chen CM, Wu YR, Tienari PJ, Singleton A: Conicting results regarding the semaphorin gene (SEMA5A) and the risk for Parkinson disease. Am J Hum Genet 2006;78:1082-1084.

-7 Farrer MJ, Haugarvoll K, Ross OA, Stone JT, Milkovic NM, Cobb SA, Whittle AJ, Lincoln SJ, Hulihan MM, Heckman MG, White LR, Aasly JO, Gibson JM, Gosal D, Lynch T, Wszolek ZK, Uitti RJ, Toft M: Genomewide association, Parkinson disease, and PARK10. Am J Hum Genet 2006;78:1084-1088.

8 Goris A, Williams-Gray CH, Foltynie T Compston DAS, Barker RA, Sawcer SJ: No evidence for association with Parkinson disease for 13 single-nucleotide polymorphisms identified by whole-genome association screening. Am J Hum Genet 2006;78:10881090.

\$ Li Y, Rowland C, Schrodi S, Laird W, Tacey $\mathrm{K}$, Ross D, Leong D, Catanese J, Sninsky J, Grupe A: A case-control association study of the 12 single-nucleotide polymorphisms implicated in Parkinson disease by a recent genome scan. Am J Hum Genet 2006;78:10901092.
10 Herbert A, Gerry NP, McQueen MB, Heid IM, Pfeufer A, Illig T, Wichmann HE, Meitinger T, Hunter D, Hu FB, Colditz G, Hinney A, Hebebrand J, Koberwitz K, Zhu X, Cooper R, Ardlie K, Lyon H, Hirschhorn JN, Laird NM, Lenburg ME, Lange C, Christman MF: A common genetic variant is associated with adult and childhood obesity. Science 2006; 312:279-283.

11 Dina C, Meyre D, Samson C, Tichet J, Marre M, Jouret B, Charles MA, Balkau B, Froguel $\mathrm{P}$ : Comment on 'a common genetic variant is associated with adult and childhood obesity'. Science 2007;315:187.

12 Loos RJF, Barroso I, O’Rahilly S, Wareham $\mathrm{NJ}$ : Comment on 'a common genetic variant is associated with adult and childhood obesity'. Science 2007;315:187.

13 Rosskopf D, Bornhorst A, Rimmbach C, Schwahn C, Kayser A, Kruger A, Tessmann G, Geissler I, Kroemer HK, Volzke H: Comment on 'a common genetic variant is associated with adult and childhood obesity'. Science 2007;315:187.

14 Wijsman EM, Amos CI: Genetic analysis of simulated oligogenic traits in nuclear and extended pedigrees: Summary of gaw10 contributions. Genet Epidemiol 1997;14:719735.

$\checkmark 15$ Silberstein M, Tzemach A, Dovgolesky N, Fishelson M, Schuster A, Geiger D: Online system for faster multipoint linkage analysis via parallel executation on thousands of personal computers. Am J Hum Genet 2006; 78 : 922-935.

16 Lange K, Sobel E: A random walk method for computing genetic location scores. Am J Hum Genet 1991;49:1320-1334.

$\checkmark 17$ Thompson EA: Monte Carlo likelihood in genetic mapping. Stat Sci 1994;9:355-366.

18 Donnelly KP: The probability that related individuals share some section of genome identical by descent. Theor Popul Biol 1983; 23:34-63.

19 Lander ES, Green P: Construction of multilocus genetic linkage maps in humans. Proc Nat Acad Sci USA 1987;84:2363-2367.

20 Heath SC: Markov chain Monte Carlo segregation and linkage analysis for oligogenic models. Am J Hum Genet 1997;61:748-760.

21 Thompson EA, Heath SC: Estimation of conditional multilocus gene identity among relatives; in Seillier-Moiseiwitsch F (ed): Statistics in Molecular Biology and Genetics: Selected Proceedings of a 1997 Joint AMSIMS-SIAM Summer Conference on Statistics in Molecular Biology, IMS Lecture Note - Monograph Series Volume 33. Hayward, CA, Institute of Mathematical Statistics, 1999, pp 95-113.
22 Thompson EA: MCMC in the analysis of genetic data on pedigrees; in Liang F, Wang JS, Kendall W (eds): Markov Chain Monte Carlo: Innovations and Applications. Singapore, World Scientific Co Pte Ltd, 2005, pp 183216.

23 MORGAN: a package for Markov chain Monte Carlo in Genetic Analysis (Version 2.8). http://www.stat.washington.edu/ thompson/Genepi/MORGAN/Morgan. shtml, 2005.

24 Wijsman EM, Rothstein JH, Thompson EA: Multipoint linkage analysis with many multiallelic or dense diallelic markers: Markov chain Monte Carlo provides practical approaches for genome scans on general pedigrees. Am J Hum Genet 2006; 79:846-858.

-25 Fischelson M, Geiger D: Optimizing exact linkage computations. J Comput Biol 2004; 11:263-275.

26 Thomas A, Gutin A, Abkevich V: Multilocus linkage analysis by blocked Gibbs sampling. Stat Comput 2000;10:259-269.

$\checkmark 27$ Sobel E, Lange K: Descent graphs in pedigree analysis: Applications to haplotyping, location scores, and marker-sharing statistics. Am J Hum Genet 1996;58:1323-1337.

28 O'Connell JR, Weeks DE: The algorithm for rapid exact multilocus linkage analysis via genotype set-recoding and fuzzy inheritance. Nature Genet 1995;11:402-408

29 Abecasis GR, Cherny SS, Cookson WO, Cardon LR: Merlin - rapid analysis of dense genetic maps using sparse gene ow trees. Nature Genet 2002;30:97-101.

30 Haldane JBS: The combination of linkage values and the calculation of distances between the loci of linked factors. J Genet 1919; 8:229-309.

- 31 Elston RC, Stewart J: A general model for the analysis of pedigree data. Hum Hered 1971; 21:523-542.

32 Sung YJ, Di Y, Fu AQ, Rothstein JH, Sieh W, Tong L, Thompson EA, Wijsman EM: Comparison of multipoint linkage analyses for quantitative traits: Parametric lod scores, variance components lod scores and bayes factors in the ceph data. Biomed Central Genet 2007; in press.

33 Baum LE, Petrie T: Statistical inference for probabilistic functions of finite state markov chains. Ann Math Stat 1966;37:1554-1563.

34 Rabiner LR: A tutorial on hidden markov models and selected applications in speech recognition. Proc IEEE 1989;77:257-286. 